\title{
GALOISIAN OBSTRUCTIONS TO INTEGRABILITY OF HAMILTONIAN SYSTEMS II*
}

\author{
JUAN J. MORALES-RUIZ ${ }^{\dagger}$ AND JEAN PIERRE RAMIS $\ddagger$
}

\begin{abstract}
By applying the results of our previous paper [19], we obtain non-integrability results for the following four Hamiltonian systems: the Bianchi IX Cosmological Model, the Sitnikov system of celestial mechanics, the Spring-Pendulum system and a generalization of the homogeneous potentials considered by Yoshida. All these systems are considered over the complex domain (complex time and complex phase space) and the integrability is considered in the Liouville sense of existence of a maximal number of first integrals in involution.
\end{abstract}

1. Introduction. This paper is the continuation of our previous papers $[19,20]$ and is devoted to the applications of the results of [19] to some models: the Bianchi IX cosmological model, the Sitnikov system of celestial mechanics, the Spring-Pendulum system and a generalization of our results in [20] about the non-integrability of homogeneous potentials.

From the differential Galois theory we will need only the algorithm of Kovacic and the theorem of Kimura about the solvability of the Riemann's hypergeometric differential equation. Then, using our results in [19], the methods proposed here are efficient, but they remain completely systematic and very elementary.

\section{Hypergeometric differential equations and Kovacic's algorithm.}

2.1. The hypergeometric equation. The hypergeometric (or Riemann) equation is the more general second order linear differential equation over the Riemann sphere with three regular singular singularities. If (using an homography) we place the singularities at $x=0,1, \infty$, then this equation is given by

$$
\begin{aligned}
\frac{d^{2} \xi}{d x^{2}} & +\left(\frac{1-\alpha-\alpha^{\prime}}{x}+\frac{1-\gamma-\gamma^{\prime}}{x-1}\right) \frac{d \xi}{d x} \\
& +\left(\frac{\alpha \alpha^{\prime}}{x^{2}}+\frac{\gamma \gamma^{\prime}}{(x-1)^{2}}+\frac{\beta \beta^{\prime}-\alpha \alpha^{\prime}-\gamma \gamma^{\prime}}{x(x-1)}\right) \xi=0,
\end{aligned}
$$

being $\left(\alpha, \alpha^{\prime}\right),\left(\gamma, \gamma^{\prime}\right),\left(\beta, \beta^{\prime}\right)$, the exponents at the singular points. These exponents must satisfy the Fuchs relation $\alpha+\alpha^{\prime}+\gamma+\gamma^{\prime}+\beta+\beta^{\prime}=1$. We denote the exponent differences by $\hat{\lambda}=\alpha-\alpha^{\prime}, \hat{\nu}=\gamma-\gamma^{\prime}$ and $\hat{\mu}=\beta-\beta^{\prime}$.

We recall that one of the reduced forms of our differential equation is

$$
\frac{d^{2} \xi}{d x^{2}}+\frac{c-(a+b+1) x}{x(1-x)} \frac{d \xi}{d x}-\frac{a b}{x(1-x)} \xi=0
$$

where $a, b, c$ are parameters, being the exponent differences $\hat{\lambda}=1-c, \hat{\nu}=c-a-b$ and $\hat{\mu}=b-a$, respectively.

Now, we recall the theorem of Kimura that gives us the necessary and sufficient conditions in order to have solvability for the hypergeometric equation (that is existence of solutions in closed form).

\footnotetext{
*Received March 1, 2000; revised April 6, 2001.

${ }^{\dagger}$ Departament de Matemàtica Aplicada II, Universitat Politècnica de Catalunya, Pau Gargallo 5, E-08028 Barcelona, Spain (morales@ma2.upc.es).

${ }^{\ddagger}$ Laboratoire Emile Picard, Université Paul Sabatier, 118, route de Narbonne, Toulouse, Cedex, France and Institut Universitaire de France (ramis@picard.ups-tlse.fr).
} 
TheOrem 1 ([11]). The identity component of the Galois group of the hypergeometric equation (2.1) is solvable if, and only if, either

(i) At least one of the four numbers $\hat{\lambda}+\hat{\mu}+\hat{\nu},-\hat{\lambda}+\hat{\mu}+\hat{\nu}, \hat{\lambda}-\hat{\mu}+\hat{\nu}, \hat{\lambda}+\hat{\mu}-\hat{\nu}$ is an odd integer, or

(ii) The numbers $\hat{\lambda}$ or $-\hat{\lambda}$, $\hat{\mu}$ or $-\hat{\mu}$ and $\hat{\nu}$ or $-\hat{\nu}$ belong (in an arbitrary order) to some of the following fifteen families

\begin{tabular}{|c|c|c|c|c|}
\hline 1 & $1 / 2+l$ & $1 / 2+m$ & arbitrary complex number & \\
\hline 2 & $1 / 2+l$ & $1 / 3+m$ & $1 / 3+q$ & \\
\hline 3 & $2 / 3+l$ & $1 / 3+m$ & $1 / 3+q$ & $l+m+q$ even \\
\hline 4 & $1 / 2+l$ & $1 / 3+m$ & $1 / 4+q$ & \\
\hline 5 & $2 / 3+l$ & $1 / 4+m$ & $1 / 4+q$ & $l+m+q$ even \\
\hline 6 & $1 / 2+l$ & $1 / 3+m$ & $1 / 5+q$ & \\
\hline 7 & $2 / 5+l$ & $1 / 3+m$ & $1 / 3+q$ & $l+m+q$ even \\
\hline 8 & $2 / 3+l$ & $1 / 5+m$ & $1 / 5+q$ & $l+m+q$ even \\
\hline 9 & $1 / 2+l$ & $2 / 5+m$ & $1 / 5+q$ & $l+m+q$ even \\
\hline 10 & $3 / 5+l$ & $1 / 3+m$ & $1 / 5+q$ & $l+m+q$ even \\
\hline 11 & $2 / 5+l$ & $2 / 5+m$ & $2 / 5+q$ & $l+m+q$ even \\
\hline 12 & $2 / 3+l$ & $1 / 3+m$ & $1 / 5+q$ & $l+m+q$ even \\
\hline 13 & $4 / 5+l$ & $1 / 5+m$ & $1 / 5+q$ & $l+m+q$ even \\
\hline 14 & $1 / 2+l$ & $2 / 5+m$ & $1 / 3+q$ & $l+m+q$ even \\
\hline 15 & $3 / 5+l$ & $2 / 5+m$ & $1 / 3+q$ & $l+m+q$ even \\
\hline
\end{tabular}

Here $l, m$ and $q$ are integers.

We recall that the Schwarz's table gives us the cases for which the Galois (and monodromy) groups are finite (i.e., the identity component of the Galois group is reduced to the identity element) and is given by fifteen families. These families are given by families $2-15$ of the table above and by the family $(1 / 2+\mathbf{Z}) \times(1 / 2+\mathbf{Z}) \times \mathbf{Q}$ (see, for instance, [22]). As this last family is already contained in family 1 of the above table, all of the Schwarz's families are, of course, contained in the above table .

2.2. Kovacic's Algorithm. The Kovacic's Algorithm gives us a procedure in order to compute a fundamental system of solutions in closed form for a second order algebraic differential equation provided this differential equation is solvable. Reciprocally, if the algorithm does not work that implies the non-solvability of the equation (see[12]). In this (necessarily brief) description we will follow the version of the algorithm given in $[9,8]$.

Given a second order linear differential equation with coefficients in $\mathbf{C}(x)$, the possibility to transform it to the so-called reduced invariant form is a classical fact.

$$
\xi^{\prime \prime}-g \xi=0
$$

being $g=g(x) \in \mathbf{C}(x)$.

We remark that in this change we introduce the exponentiation of a quadrature. Then the solvability of the original equation is equivalent to the solvability of the above equation (although, in general, the Galois groups are not the same).

The algorithm is based on the following two general facts:

(A) The classification of the algebraic subgroups of $S L(2, \mathbf{C})$ (see, for instance, [19], Proposition 1 and references therein) (the Galois group of the equation (2.3) is contained in $S L(2, \mathbf{C}))$. 
(B) The well-known transformation to a Riccati Equation (by the change $v=\xi^{\prime} / \xi$ ),

$$
v^{\prime}=g-v^{2}
$$

Then the differential equation (2.3) is solvable, if and only if, the equation (2.4) has an algebraic solution. The key point now is that the degree $n$ of the associated minimal polynomial $Q(v)$ (with coefficients in $\mathbf{C}(x)$ ) belongs to the set

$$
L_{\max }=\{1,2,4,6,12\} .
$$

The determination of the set $L$ of possible values for $n$, is the First Step of the algorithm. We remark that for $n=4, n=6$ and $n=12$, the Galois group of (2.3) is finite (hence these values are related to the cristalographic groups). The two other steps of the algorithm (Second Step and Third Step) are devoted to the computation of the polynomial $Q(v)$ (if it exist). If the algorithm does not work (i.e., if the equation (2.4) has not algebraic

solution) then the equation (2.3) is not solvable and its Galois group is $S L(2, \mathbf{C})$.

Now we will describe the algorithm.

Let $g(x)=\frac{s(x)}{t(x}$, being $s(x), t(x)$ relatively prime polynomials, and $t(x)$ monic. We define the following function $h$ on the set $L_{\max }=\{1,2,4,6,12\}, h(1)=1, h(2)=4$, $h(4)=h(6)=h(12)=12$

\section{First Step} mials.

If $t(x)=1$ we put $m=0$, else we factorize $t(x)$ in monic relatively prime polyno-

1.1. Let $\Gamma^{\prime}$ be the set of roots of $t(x)$ (i.e., the singular points at the finite complex plane) and let $\Gamma=\Gamma^{\prime} \cup \infty$ be the set of singular points. Then the order at a singular point $c \in \Gamma^{\prime}$ is, as usual, $o(c)=i$ if $c$ is root of multiplicity $i$ of $t(x)$ and the order at infinity is defined by $o(\infty)=\max (0,4+\operatorname{deg}(s)-\operatorname{deg}(t))$. Then we write $m^{+}$for the maximum value of the order that appears at the singular points in $\Gamma$ and $\Gamma_{i}$ is the set of singular singular points of order $i \leq m^{+}$.

1.2. If $m^{+} \geq 2$ then we write $\gamma_{2}=\operatorname{card}\left(\Gamma_{2}\right)$, else $\gamma_{2}=0$. Then we compute

$$
\gamma=\gamma_{2}+\operatorname{card}\left(\cup \underset{\substack{k \text { odd } \\ 3 \leq k \leq m+}}{ } \Gamma_{k}\right)
$$

1.3. For the singular points of order one or two, $c \in \Gamma_{1} \cup \Gamma_{2}$ one compute the principal parts of $g$ :

$$
g(x)=\alpha_{c}(x-c)^{-2}+O\left((x-c)^{-1}\right),
$$

if $c \in \Gamma^{\prime}$, and

$$
g(x)=\alpha_{\infty} x^{-2}+O\left(x^{-3}\right)
$$

for the point at infinite.

1.4. We define the subset $L^{\prime}$ (of possible values for the degree of the minimal polynomial $Q(v))$ as $\{1\} \subset L^{\prime}$ if $\gamma=\gamma_{2},\{2\} \subset L^{\prime}$ if $\gamma \geq 2$ and $\{4,6,12\} \subset L^{\prime}$ if $m^{+} \leq 2$.

1.5. We have the three following mutually exclusive cases: 
1.5.1. If $m^{+}>2$, then $L=L^{\prime}$.

1.5.2. If $m^{+} \leq 2$ and for any $c \in \Gamma, \sqrt{1+4 \alpha_{c}} \in \mathbf{Q}, \sum_{c \in \Gamma^{\prime}} \beta_{c}=0$, then $L=L^{\prime}$.

1.5.3. If cases 1.5.1 and 1.5.2 do not hold then $L=L^{\prime}-\{4,6,12\}$.

1.6. If $L=\emptyset$, then equation (2.3) is non-integrable with Galois group $S L(2, \mathbf{C})$, else one writes $n$ for the minimum value in $L$.

For the Second and the Third Step of the algorithm we consider fixed the value of $n$.

\section{Second Step}

2.1. If $\infty$ has order 0 we write the set

$$
E_{\infty}=\left\{0, \frac{h(n)}{n}, 2 \frac{h(n)}{n}, 3 \frac{h(n)}{n}, \ldots, n \frac{h(n)}{n}\right\} .
$$

2.2. If $c$ has order 1 , then $E_{c}=\{h(n)\}$.

2.3. If $n=1$ and for each $c$ of order 2, we define

$$
E_{c}=\left\{\frac{1}{2}\left(1+\sqrt{1+4 \alpha_{c}}\right), \frac{1}{2}\left(1-\sqrt{1+4 \alpha_{c}}\right)\right\} .
$$

2.4. If $n \geq 2$, for each $c$ of order 2 , we define

$$
E_{c}=\mathbf{Z} \cap\left\{\frac{h(n)}{2}\left(1-\sqrt{1+4 \alpha_{c}}\right)+\frac{h(n)}{n} k \sqrt{1+4 \alpha_{c}}: k=0,1, \ldots, n\right\}
$$

2.5. If $n=1$, for each singular point of even order $2 \nu$, we compute the numbers $\alpha_{c}$ (different from the $\alpha$ computed in 1.3) and $\beta_{c}$ defined (up to a sign) by the following conditions:

2.5.1. If $c \in \Gamma^{\prime}$,

$$
g(x)=\left\{\alpha_{c}\left((x-c)^{-\nu}\right)+\sum_{i=2}^{\nu-1} \mu_{i, 2}\left((x-c)^{-i}\right)\right\}^{2}+\beta_{c}\left((x-c)^{-\nu-1}\right)+O\left((x-c)^{-\nu}\right),
$$

and we write

$$
\sqrt{g}_{c}:=\alpha_{c}\left((x-c)^{-\nu}\right)+\sum_{i=2}^{\nu-1} \mu_{i, 2}\left((x-c)^{-i}\right)
$$

2.5.2. If $c=\infty$,

$$
g(x)=\left\{\alpha_{\infty}\left(x^{\nu-2}\right)+\sum_{i=0}^{\nu-3} \mu_{i, \infty} x^{i}\right\}^{2}-\beta_{\infty} x^{\nu-3}+O\left(x^{\nu-4}\right),
$$

and we write

$$
\sqrt{g}_{\infty}:=\alpha_{\infty}\left(x^{\nu-2}\right)+\sum_{i=0}^{\nu-3} \mu_{i, \infty} x^{i}
$$


Then

$$
E_{c}=\left\{\frac{1}{2}\left(\nu+\epsilon \frac{\beta_{c}}{\alpha_{c}}\right): \epsilon= \pm 1\right\}
$$

and the sign on $E_{c}$ is defined by

$$
\operatorname{sign}\left(\frac{1}{2}\left(\nu+\epsilon \frac{\beta_{c}}{\alpha_{c}}\right)=\epsilon,\right.
$$

being +1 if $\beta_{c}=0$.

2.6. If $n=2$, for each $c$ of order $\nu$, with $\nu \geq 3$, we write $E_{c}=\{\nu\}$.

\section{Third Step}

3.1. For $n$ fixed, we try to obtain elements $\mathbf{e}=\left(e_{c}\right)_{c \in \Gamma}$ which belong to the cartesian product $\prod_{c \in \Gamma} E_{c}$, such that

(i) $d(\mathbf{e}):=n-\frac{n}{h(n)} \sum_{c \in \Gamma} e_{c}$ is a natural number, and

(ii) If $n=2$ then in e there is at least one odd number.

If no element $\mathbf{e}$ is obtained, then we select the next value in $L$ and go to the

Second Step, else $n$ is the maximum value in $L$ and the Galois group is $S L(2, \mathbf{C})$ (i.e., the equation (2.3) is not solvable).

3.2. For each family e as above, we try to obtain a rational function $Q$ and a polynomial $P$, such that

(i)

$$
Q=\frac{n}{h(n)} \sum_{c \in \Gamma^{\prime}} \frac{e_{c}}{x-c}+\delta_{n 1} \sum_{c \in \cup_{\nu>1} \Gamma_{2 \nu}} \operatorname{sign}\left(e_{c}\right) \sqrt{g}_{c}
$$

being $\delta_{n 1}$ the delta of Kronecker.

(ii) $P$ is a polynomial of degree $d(\mathbf{e})$ and its coefficients are found as a solution of the (in general, overdetermined) system of equations

$$
\begin{aligned}
& P_{-1}=0 \\
& P_{i-1}=-\left(P_{i}\right)^{\prime}-Q P_{i}-(n-i)(i+1) g P_{i+1}, n \geq i \geq 0 \\
& P_{n}=-P .
\end{aligned}
$$

If a pair $(P, Q)$ as above is found, then equation (2.3) is solvable and the Riccati Equation (2.4) has an algebraic solution $v$ given by any root $v$ of the equation

$$
\sum_{i=0}^{n} \frac{P_{i}}{(n-1) !} v^{i}=0
$$

If no pair as above is found we take the next value in $L$ and we go to the Second Step. If $n$ is the greatest value in $L$ then the equation (2.3) is not solvable and the Galois group is $S L(2, \mathbf{C})$.

We notice that a remarkable simplification of the above algorithm, for an irreducible differential equation, was obtained in [27] and that an algorithm for third order differential equations is given in $[24,25]$. 
3. Non-integrability Theorems. We consider a complex analytic symplectic manifold $M$ of dimension $2 n$ and let $X_{H}$ be a holomorphic Hamiltonian system defined on it. Let $\Gamma$ be the (abstract) connected Riemann surface corresponding to a maximal integral curve $i(\Gamma)$ defined by $z=z(t)$ (which is not reduced to an equilibrium point) of $X_{H}$. Then we can obtain the variational equations $(V E)$

$$
\dot{\eta}=X_{H}^{\prime}(z(t)) \eta
$$

along $\Gamma$, and we can also obtain the normal variational equations $(N V E)$ along $\Gamma$. These variational equations are holomorphic (resp. meromorphic) connections over $\Gamma$ and it is possible to interpret them as meromorphic linear differential equations over Г. (For more details see [19].)

In the above situation, we prove in [19] the following result

THEOREM 2. Assume that there are $n$ first integrals of $X_{H}$ which are meromorphic, in involution and independent in a neighbourhood $U$ of the curve $i(\Gamma)$ in $M$ (more precisely we suppose that they are not necessarily independent on $i(\Gamma)$ itself, only over an open dense subset of $U$ ). Then the identity component of the Galois group of the VE (resp. (NVE)) is an abelian subgroup of the symplectic group.

In particular, the $V E$ (and the $N V E$ ) are solvable in the sense of the Differential Galois Theory. To be more precise, the Picard-Vessiot extension of the $V E$ (and the $N V E$ ) is obtained by (a combination of) quadratures, exponential of quadratures and algebraic functions. We recall that this is the case if, and only if, the identity component of the Galois group is solvable (see, for instance [3, 10, 16, 23]).

In the above theorem the differential Galois group $G$ is computed relatively to the differential field $k_{\Gamma}$ of meromorphic functions over $\Gamma$. Then it is well known that $G$ is the Zariski closure in the general linear group of the monodromy group of the variational equation. (That is $G$ the smallest complex algebraic linear group containing the monodromy group.)

In some interesting cases it is possible to add to our Riemann surface $\Gamma$ a finite number of points corresponding to equilibrium points belonging to the closure of $i(\Gamma)$ in $M$. We get $i(\Gamma) \subset \underline{\Gamma} \subset M$ and $\Gamma \subset \bar{\Gamma}$ and we suppose that $\underline{\Gamma}$ is a closed analytic curve (not necessarily smooth) of $M$ and that $\bar{\Gamma}$ is a connected Riemann surface.

Then in some cases (as in the study of the Bianchi IX Cosmological Model that we will do below) it is also useful to add to the Riemann surface $\bar{\Gamma}$ a finite number of points corresponding to points at infinity of $\underline{\Gamma}$ ( this new Riemann surface is denoted by $\bar{\Gamma}^{\prime}$ ). More precisely we suppose that the symplectic manifold $M$ is contained in a connected manifold $M^{\prime}$, such that $M_{\infty}=M^{\prime}-M$ is an analytic (non necessarily smooth) hypersurface in $M^{\prime}$ (the hypersurce at infinity), and that the holomorphic symplectic form $\Omega$ over $M$ extends as a closed meromorphic form $\Omega^{\prime}$ over $M^{\prime}$ [19]. Then we get $\underline{\Gamma} \subset \underline{\Gamma}^{\prime} \subset M^{\prime}$ and $\bar{\Gamma} \subset \bar{\Gamma}^{\prime}$. We suppose that $\underline{\Gamma}^{\prime}$ is a closed analytic curve (not necessarily smooth) of $M^{\prime}$ and that $\bar{\Gamma}^{\prime}$ is a connected Riemann surface. Then the connection of the $V E$ extends as a meromorphic connection over $\bar{\Gamma}$ (resp. $\bar{\Gamma}^{\prime}$ ) and we can compute the differential Galois group $\bar{G}$ (resp. $\bar{G}^{\prime}$ ) of the $V E$ (or the $N V E$ ) relatively to the differential field $k_{\bar{\Gamma}}$ (resp. $k_{\bar{\Gamma}^{\prime}}$ ) of meromorphic functions over $\bar{\Gamma}$ (resp. $\left.\bar{\Gamma}^{\prime}\right)$.

Then $G \subset \bar{G} \subset \bar{G}^{\prime}$ but in general these inclusions are strict. However when the extended connection of the $V E$ over $\bar{\Gamma}$ (resp. $\bar{\Gamma}^{\prime}$ ) is Fuchsian (that is when its singular points are regular singular), then we have $G=\bar{G}$ (resp. $G=\bar{G}^{\prime}$ ). It will be the case 
below in the present paper except in the study of the Bianchi IX model. For this study we need a modified version of the above theorem ([19]).

THEOREM 3. We assume that we have a finite set of equilibrium points and of points at infinity. We assume that there are $n$ first integrals of $X_{H}$ which are meromorphic, in involution and independent in a neighbourhood $U$ of the curve $\underline{\Gamma}^{\prime}$ in $M^{\prime}$ (more precisely we suppose that they are not necessarily independent on $i(\bar{\Gamma})$ itself, only over an open dense subset of $U$ ). Then the identity component of the Galois group $\bar{G}^{\prime}$ of the VE (resp. (NVE)) over the differential field $k_{\bar{\Gamma}^{\prime}}$ is an abelian subgroup of the symplectic group.

In the situation of this theorem the identity component of $G$ is of course also abelian, but in general this identity component is smaller than the identity component of $\bar{\Gamma}^{\prime}$, therefore Theorem 3 give a stronger obstruction than Theorem 2. Moreover it is in general easier to get algebraic information about $\bar{G}^{\prime}$ than to get algebraic information about $G$. In the case of Bianchi IX cosmological model, we will see that the identity component of $\bar{G}^{\prime}$ is not abelian, however the identity component of $G$ could be abelian: it is an open problem. We insist also upon the following fact: if using Theorem 3 we have proved that our system is not integrable in a neighbourhood of $\Gamma^{\prime}$ in $M^{\prime}$, the existence of a system of $n$ first integrals which are independent, in involution and meromorphic over $M$, but with a non meromorphic growth at infinity, remains possible.

In the examples below the Riemann surface $\bar{\Gamma}$ in the Fuchsian case (resp. $\bar{\Gamma}^{\prime}$ in the case of an irregular singularity at infinity) will be compact and we will have a purely algebraic systematic method to decide if the identity component of $G$ (resp. $\bar{G}^{\prime}$ )is not abelian (in fact non solvable). It will be based upon Kovacic's algorithm and Kimura's result about hypergeometric differential equations.

\section{The Bianchi IX Cosmological Model.}

4.1. The Model. The Bianchi IX Cosmological model is a dynamical system given by the equations in "logarithmic" time $([14])$

$$
\begin{aligned}
& \frac{d^{2} \log x_{1}}{d t^{2}}=\left(x_{2}-x_{3}\right)^{2}-x_{1}^{2}, \\
& \frac{d^{2} \log x_{2}}{d t^{2}}=\left(x_{3}-x_{1}\right)^{2}-x_{2}^{2}, \\
& \frac{d^{2} \log x_{3}}{d t^{2}}=\left(x_{1}-x_{2}\right)^{2}-x_{3}^{2},
\end{aligned}
$$

with the energy constraint (from physical considerations)

$$
E=-\left(\frac{\dot{x}_{1}}{x_{1}} \frac{\dot{x}_{2}}{x_{2}}+\frac{\dot{x}_{2}}{x_{2}} \frac{\dot{x}_{3}}{x_{3}}+\frac{\dot{x}_{3}}{x_{3}} \frac{\dot{x}_{1}}{x_{1}}\right)+x_{1}^{2}+x_{2}^{2}+x_{3}^{2}-2\left(x_{1} x_{2}+x_{2} x_{3}+x_{3} x_{1}\right)=0
$$

So, we get a dynamical system of dimension five on the zero level energy manifold $M_{0}$.

In fact this system is a Hamiltonian system with position variables $x_{1}, x_{2}, x_{3}$ and conjugate moments given by

$$
y_{1}=-\frac{1}{x_{1}} \frac{d}{d t} \log \left(x_{2} x_{3}\right),
$$




$$
\begin{aligned}
& y_{2}=-\frac{1}{x_{2}} \frac{d}{d t} \log \left(x_{1} x_{3}\right), \\
& y_{3}=-\frac{1}{x_{3}} \frac{d}{d t} \log \left(x_{1} x_{2}\right) .
\end{aligned}
$$

And the energy become the Hamiltonian

$$
\begin{gathered}
H=\frac{1}{4}\left(x_{1}^{2} y_{1}^{2}+x_{2}^{2} y_{2}^{2}+x_{3}^{2} y_{3}^{2}-2 x_{1} x_{2} y_{1} y_{2}-2 x_{2} x_{3} y_{2} y_{3}-2 x_{1} x_{3} y_{1} y_{3}\right) \\
+x_{1}^{2}+x_{2}^{2}+x_{3}^{2}-2 x_{1} x_{2}-2 x_{2} x_{3}-2 x_{1} x_{3}=0
\end{gathered}
$$

This system is studied from a real dynamical point of view in $[14,4,18,7]$ (the real configuration space for this system is given by $\left.x_{i}>0, i=1,2,3\right)$. It is proved in [7] that it is a locally integrable Hamiltonian system (for a precise definition of what that means, see the above reference) on the open real physical phase space. As it is not recurrent in the real open phase space, it is necessary to compactify this physical phase space in order to have recurrence and then it is proved in [4] (p. 54-76) that the behaviour of the system is very complicated in this extended real phase space (this is also studied in a more heuristic way in [14], p. 477-484) with oscillations around the gravitational collapse (the gravitational collapse is given by the points in the phase space satisfying $x_{1} x_{2} x_{3}=0$ ). It seems that some new insight of the chaotic real behaviour of the Bianchi IX model was recently given by Cornish and Levin in the preprint [5]. This and other additional recent references on the dynamics of Bianchi IX are given in [2].

The complex dynamical point of view (with complex phase space and complex time) on this problem appeared more recently. In this direction the paper [15] is devoted to the fact that this dynamical system do not pass the Painlevé's test (this method in order to test non integrability started with the Kowaleski's analysis of the rigid body [13]), i.e., that the only movable singularities (of the solutions with respect to the time parametrization) are poles. (Some people allow also the existence of movable essential singularities.) The authors use the variational equations along a family of particular solutions found by Taub ([26]), and they show that these variational equations have an irregular singular (formally ramified) point and hence that the solutions of the corresponding system have essential singularities related to the Taub family. We notice that from the existence of an irregular singularity it follows immediately that the general solution of the system (with five parameters) is not meromorphic in time. From the above considerations, it is clear that the Bianchi IX model is a subject of polemic: apparently contradictory results were obtained. A part of this puzzle is clarified by the following two remarks.

Firstly there are several degrees of integrability in Liouville sense for a Hamiltonian system: real integrability (given by differentiable or analytical real first integrals), algebraic integrability (the Liouville tori become real subvarieties of abelian varieties), integrability by algebraic (or more generally rational) first integrals, by holomorphic (or more generally meromorphic) first integrals. Moreover the relations between Painlevé's test and integrability in Liouville sense is not so clear.

A second elementary but important remark is that, if we are studying some kind of integrability, then the allowed changes of variables (i.e., canonical transformations) must belong to the same precise class. For instance, if we are studying the integrability of a system by algebraic first integrals (or if this system has the Painlevé property), then changes of variables must have the same degree of regularity. So a change of variables that introduces essential singularities in the phase variables (respectively in 
the time) is not allowed, because the above integrability (respectively the Painlevé's property) is not preserved by this change. Then the Bianchi IX cosmological model is a good "laboratory" in order to understand the interplay between the several concepts of integrability used by different authors.

In this section we shall prove the non-integrability of the Bianchi IX model (with the Hamiltonian given by $H$ above) by rational first integrals.

4.2. Non-integrability. Our proof of the non-integrability relies on the study of the variational equations along particular solutions of the Taub family.

As Taub noticed, the subspace $x_{2}=x_{3}\left(\dot{x}_{2}=\dot{x}_{3}\right)$ is invariant by the flow of the system, and the reduction to this invariant four dimensional space (three dimensional if we consider the restriction of the system to the restricted manifold $M_{0}$ ) is completely integrable (i.e. it is an integrable subsystem) and the solutions can be calculated explicitly ([26], p. 481). From the Taub family of solutions we select the particular ones

$$
x_{1}=\frac{2 k}{\cosh (2 k t)}, \quad x_{2}=x_{3}=\frac{k \cosh (2 k t)}{2 \cosh ^{2}(k t)},
$$

being $k$ a parameter. This particular integral curve (for a fixed value of $k$ ) is our integral curve $i(\Gamma)$, along which we compute the $V E$. We remark that $i(\Gamma) \subset M_{0}$ (energy constraint). Furthermore $\bar{\Gamma}^{\prime}$ is here the Riemann sphere $\mathbf{P}^{1}$, since it admits a global rational parametrization in the variable $z:=\tanh k t$ : we can write $x_{1}, x_{2}=$ $x_{3}, \dot{x}_{1}, \dot{x}_{2}=\dot{x}_{3}$ as rational functions of $z$,

$$
\begin{gathered}
\left.x_{1}=\frac{2 k\left(1-z^{2}\right)}{1+z^{2}}, \quad x_{2}=x_{3}=\frac{k\left(1+z^{2}\right)}{2}\right), \\
\dot{x}_{1}=-\frac{8 k^{2} z\left(1-z^{2}\right)}{\left(1+z^{2}\right)^{2}}, \quad \dot{x}_{2}=\dot{x}_{3}=k z\left(1-z^{2}\right) .
\end{gathered}
$$

The relevant part of the $V E$ is given by the Normal Variational Equation $(N V E)$ (transversal to the invariant space $x_{2}=x_{3}, \dot{x}_{2}=\dot{x}_{3}$ ) obtained in [15] (we recall that the tangential part of the $V E$ is always solvable, see [19])

$$
\ddot{\alpha}-2\left(x_{1} x_{2}-2 x_{2}^{2}\right) \alpha=0 .
$$

Now, as it is noted in [15], it is possible to write the $N V E$ over the Riemann sphere $\mathbf{P}^{1}$ (i.e., with rational coefficients) by the change $x:=z^{2}=\tanh ^{2}(k t)$. We write the obtained equation in its invariant reduced form (i.e., without the first derivative term in the standard way) using the new independent variable $\eta$

$$
\ddot{\eta}+\left(\frac{1}{4} \frac{1}{x-1}+\frac{5}{4} \frac{1}{(x-1)^{2}}+\frac{3}{16} \frac{1}{x^{2}}\right) \eta=0 .
$$

We remark that in the above equation the parameter $k$ is missing.

The above equation has regular singularities at $x=0,1$ and an irregular singularity at $x=\infty$ (it is a confluent Heun's Equation). The dynamical meaning of the singular points is the following. The natural parametrization of the Riemann surface $\bar{\Gamma}^{\prime} \approx \mathbf{P}^{1}$ is obtained using the variable $z$, being $x=z^{2}$. Then by the double covering $\mathbf{P}^{1} \longrightarrow \mathbf{P}^{1}, z \mapsto x$, from the points $x=0,1, \infty$, we get the four points $z=0, \pm 1, \infty$ of $\bar{\Gamma}^{\prime}$. It is a direct consequence of the rational parametrization of $\bar{\Gamma}^{\prime}$ by the parameter $z$, obtained above, that $z=0, \pm 1$ (hence $x=0,1$ ) are equilibrium 
points, with $x_{1}(0)=2 k, x_{2}(0)=x_{3}(0)=k / 2, \dot{x}_{i}(0)=0, i=1,2,3$ and $x_{1}( \pm 1)=0$, $x_{2}( \pm 1)=x_{3}( \pm 1)=k, x_{i}( \pm 1)=0, i=1,2,3$, respectively. The point $x=\infty$ corresponds to the point at infinity $z=\infty$ of the phase curve $i(\Gamma)$.

We remark also that the points $z= \pm 1$ (i.e., $x=1$ ) correspond to the physical gravitational collapse, because

$$
x_{1} x_{2} x_{3}=\frac{k^{3}}{2}\left(1-z^{4}\right)=\frac{k^{3}}{2}\left(1-x^{2}\right) .
$$

Now, we can apply Kovacic's algorithm.

Let

$$
g(x)=-\left(\frac{1}{4} \frac{1}{x-1}+\frac{5}{4} \frac{1}{(x-1)^{2}}+\frac{3}{16} \frac{1}{x^{2}}\right):=\frac{s(x)}{t(x)},
$$

with $t$ a monic polynomial. Then the algorithm is divided in three steps:

\section{First Step}

1.1. The sets $\Gamma$ and $\Gamma^{\prime}$ are

$$
\Gamma=\{0,1, \infty\}, \quad \Gamma^{\prime}=\{0,1\}
$$

with orders $o(0)=o(1)=2, o(\infty)=\max (0,4+\operatorname{deg} s-$ degt $)=3$ and define $m^{+}:=$ $\max (m, o(\infty))=\max (2, o(\infty))=3\left(m\right.$ is the cardinal of $\left.\Gamma^{\prime}\right)$.

Then the set of singular points is classificated by the order:

$$
\Gamma_{2}=\{0,1\}, \quad \Gamma_{3}=\{\infty\} .
$$

1.2. As $m^{+}>2$, we get $\gamma_{2}=2$, and $\gamma:=\gamma_{2}+\# \Gamma_{3}=3$.

1.3. $\alpha_{0}=-\frac{3}{16}, \alpha_{1}=-\frac{5}{4}$.

1.4. $L=\{2\}, n=2$.

\section{Second Step}

2.4. As $n=2 \geq 2, h(2)=4$ and $E_{0}==\mathbf{Z} \cap\{1,2,3\}=\{1,2,3\}, E_{1}=\mathbf{Z} \cap\{2(1-$ $\sqrt{-4}), \quad 2, \quad 2(1+\sqrt{-4})\}=\{2\}$.

2.6. $E_{\infty}=\{3\}$

\section{Third Step}

3.1. If $e_{1}=(1,2,3), e_{2}=(2,2,3), e_{3}=(3,2,3)$ (the elements of $\left.E_{0} \times E_{1} \times E_{\infty}\right)$, then the numbers $d\left(e_{i}\right), i=1,2,3$ are not natural numbers. Hence the Galois group of the equation (4.3) is $S L(2, \mathbf{C})$. This equation is not solvable and the NVE is not solvable too.

By Theorem 3, we have proved the following result (the irregular point of the $V E$ is at $\infty$ in the phase space).

Proposition 1. The Bianchi IX Cosmological Model considered as a complex Hamiltonian system, given by the Hamilton function $H$ introduced above, is not completely integrable with rational first integrals. 
We note that although for obtaining the above result we did not work in canonical coordinates (i.e., in a canonical frame for the $V E$ ), the result is in fact independent of this, because our formulation of the non-integrability theorems of section 3 is completely intrinsic (see [19]).

\section{The Sitnikov's Three Body Problem.}

5.1. The Model. The Sitnikov system is a restricted three body problem given by a very symmetrical configuration: the primaries with equal masses $m$ move in ellipses of excentricity $e$ in the $X Y$ plane around their center of masses $O$ and the third infinitesimal body moves along the axis $O Z$ perpendicular to the plane where the primaries move ([21]). We take, as usual, the normalization of units in such a way that $m=1 / 2$, the period of the primaries is $2 \pi$ and the gravitational constant is equal to one.

Then the equation of motion of the third body is given by

$$
\ddot{z}+\frac{z}{\left(z^{2}+r(t)^{2}\right)^{3 / 2}}=0,
$$

being $r(t)$ the distance of one of the primaries to the center of masses $O$.

In his book ([21]), Moser showed the non-integrability (and in fact the chaotic behaviour of this system) by proving that it contains the Bernouilli shift as a subsystem (in the real domain of the phase space). Here we will give an alternative proof of the analytical non-integrability of this system in the complex domain.

We follow [17] and we choose as a new time the excentric anomaly $\tau$. The transformation is given by the Kepler equation

$$
t=\tau-e \sin \tau
$$

Then the equation (5.1) is transformed in

$$
\begin{gathered}
\frac{d z}{d \tau}=(1-e \cos \tau) v \\
\frac{d v}{d \tau}=-\frac{(1-e \cos \tau) z}{\left(z^{2}+r(\tau)^{2}\right)^{3 / 2}}
\end{gathered}
$$

being

$$
r(\tau)=\frac{1}{2}(1-e \cos \tau) .
$$

5.2. Non-integrability. As the particular integral curve $\Gamma$ (or more precisely $i(\Gamma))$ we take the triple collision orbit: $e=1, r=1 / 2(1-\cos \tau, z=v=0$. The NVE along $\Gamma$ is given by

$$
\frac{d^{2} \xi}{d \tau^{2}}-\frac{\sin \tau}{1-\cos \tau} \frac{d \xi}{d \tau}+\frac{8}{1-\cos \tau} \xi=0
$$
mation

Now, in order to get an equation over the Riemann Sphere, we do the transfor-

$$
x=\frac{\cos \tau}{2}+1
$$

We obtain

$$
\frac{d^{2} \xi}{d x^{2}}+\left(\frac{1 / 2}{x}-\frac{1 / 2}{x-1} \frac{d \xi}{d x}+4\left(\frac{1}{(x-1)^{2}}-\frac{1}{x-1}+\frac{1}{x}\right) \xi=0\right.
$$


The above equation is a Riemann's (or generalized hypergeometric) equation with three regular singular singularities at $x=0, x=1$ and $x=\infty$. The triple collision corresponds to the singular point $x=1$ and the point $x=0$ corresponds to a branching point of the double covering defined by the above change of variables $x \mapsto \tau$ (geometricaly these two points corresponds to the two vertexes of the degenerated ellipse with $e=1$ ). We remark that by the Theorem 5 of [19], the identity component of the Galois Group of the equation (5.2) is the same that the identity component of the Galois Group of equation (5.3).

For the equation (5.3), the difference of exponents at $x=0, x=1, x=\infty$ is (respectively) $\lambda=1 / 2, \mu=i / 2 \sqrt{55}$ and $\nu=1$.

It is very easy to check the conditions $(i)$ or $(i i)$ of Kimura's theorem (Theorem 1) for the equation (5.3): as it is not finite (the exponents at the point $x=1$ are not rational numbers) we only need to check $(i)$ and $(i i)$, case 1 . In a direct way we get the non-solvability of the identity component of the Galois Group. In particular, this identity component is not abelian. Hence, we have obtained the following result.

Proposition 2. In a neighbourhood of the triple collision orbit, the Sitnikov Three Body Problem is not completely integrable with meromorphic first integrals.

6. The Spring-Pendulum . The spring-pendulum system is a Hamiltonian system defined in the suitable coordinates by the Hamiltonian (see $[1,6]$ and references therein)

$$
H=\frac{1}{2}\left(y_{1}^{2}+\frac{y_{2}^{2}}{x_{1}^{2}}\right)+\frac{1}{2} x_{1}^{2}+x_{1}\left(\lambda-1-\lambda \cos x_{2}\right)
$$

It represents a particle of mass $m$ attached to a massless spring of length $l$ (in equilibrium) and elastic constant $k$ in a constant gravitational field of strength $g$, then

$$
\lambda=\frac{m g}{m g+k l} .
$$

We remark that for the physical model $0 \leq \lambda \leq 1$.

We can expand the Hamilton function in powers of the variable $x_{2}$ and we get

$$
H=\frac{1}{2}\left(y_{1}^{2}+\frac{y_{2}^{2}}{x_{1}^{2}}\right)+\frac{1}{2}=x_{1}^{2}-x_{1}+\frac{\lambda}{2} x_{1} x_{2}^{2}+O\left(x_{2}^{3}\right) .
$$

Then the plane $x_{2}=y_{2}=0$ is invariant and, in the quoted above reference [6], the authors studied the $N V E$ along the integral curves contained in this plane. In fact they made a covering $z:=\frac{1}{2} x_{1} \mapsto t$ and they got an algebraic form $A N V E$ of the $N V E$. This $A N V E$ is a hypergeometric (or Riemann) equation:

$$
\frac{d^{2} \xi}{d z^{2}}+\left(\frac{5 / 2}{z}+\frac{1 / 2}{z-1}\right) \frac{d \xi}{d z}+\left(\frac{\lambda / 2}{z^{2}}+\frac{\lambda / 2}{z(1-z)}\right) \xi=0
$$

We recall that the identity components of the Galois group of the $N V E$ and of the Galois group of the equation (6.2) are the same (up to isomorphism) (Theorem 5 of $[19])$.

Then, in the reference [6], it is proved that a necessary condition for integrability is that

$$
\lambda=\frac{1}{8}\left(9-q^{2}\right)
$$


being $q$ a rational number.

In order to apply Kimura's theorem (Theorem 1), we need to compute the difference of exponents. They are $\hat{\lambda}=\frac{1}{2} \sqrt{9-8 \lambda}, \hat{\mu}=\frac{1}{2}$ and $\hat{\nu}=2$.

Then by Kimura's theorem (and Theorem 2) we get the following result (we leave the details to the reader as an exercise).

Proposition 3. The Hamiltonian system defined by (6.1) is non-integrable with a meromorphic first integral, except if $\lambda=\frac{1}{2}(2-p(p+1))$, being $p$ an integer.

We observe that for $\lambda$ in the physical region $0 \leq \lambda \leq 1$, we get non-integrability if $0<\lambda<1$. The cases $\lambda=0,1$ are separable (the case $\lambda=1$, is expressed in [1] in cartesian coordinates). Then we closed the integrability problem for the physical model.

7. A generalization of homogeneous potentials. As a last example we will generalize our non-integrability results about the model of homogeneous potentials ([20]), to Hamiltonian systems of the type

$$
H=T+V
$$

being $V$, as before, an homogeneous function of degree $k$ of the positions $x_{1}, \ldots, x_{n}$, but $T$ is now also an homogeneous function of degree $m$ of the momenta $y_{1}, \ldots, y_{n}$ (in the paper [20], we studied a particular case of this with $m=2$ ).

Working as in the case of homogeneous potentials $(m=2)$, Yoshida showed that, under some restrictions on the homogeneous functions $T$ and $V$, it is also possible to obtain a direct sum of hypergeometric equations such that the $N V E$ along an algebraic curve (which is contained in an invariant plane) appears as a pull-back of this direct sum by a finite covering of the Riemann sphere. We call it the algebraic normal variational equations: $A N V E=A N V E_{1} \oplus A N V E_{2} \oplus \ldots \oplus A N V E_{n-1}$. Each $A V N E_{i}$ is a hypergeometric equation (2.2), with parameters

$$
a+b=1 / m-1 / k, a b=-\frac{\lambda_{i}}{m k}, c=1-\frac{1}{k},
$$

here the Yoshida coefficients, $\lambda_{i}$, are the products of the eigenvalues of the Hessian of $T$ and of the corresponding eigenvalues of the Hessian of $V$ (obtained by simultaneous diagonalization of both quadratic forms). As usual we will not consider the parameter $\lambda_{n}$ corresponding to the tangential variational equation (see [28] for the details).

Then by application of Kimura's theorem (and of Theorem 2 ) we can obtain a generalization of Theorem 3 of [20]. As the explicit list of the values of the parameters $\left(m, k, \lambda_{i}\right)$ compatible with integrability is here very long, it seems better to check directly Kimura's theorem for the particular system under study. Therefore we will give the main result in a indirect way.

Proposition 4. If the Hamiltonian system given by the Hamiltonian (7.1) is completely integrable with meromorphic first integrals, then for each $i=1,2, \ldots n-1$, the values $\left(m, k, \lambda_{i}\right)$ are such that the corresponding hypergeometric equation must satisfy some of the conditions (i) or (ii) of Kimura's theorem (Theorem 1).

In his paper Yoshida considered the two degrees of freedom Hamiltonian

$$
H=F\left(y_{1}, y_{2}\right)+F\left(x_{1}, x_{2}\right),
$$


with

$$
F\left(z_{1}, z_{2}\right):=\frac{1}{4}\left(z_{1}^{4}+z_{2}^{4}\right)+\frac{e}{2} z_{1}^{2} z_{2}^{2}
$$

This Hamiltonian has two invariant planes with an $A N V E$ of hypergeometric type and with Yoshida's coefficient $\lambda$ equal to

$$
e^{2}
$$

and

$$
\left(\frac{3-e}{1+e}\right)^{2}
$$

respectively (see [28] for details).

Now we introduce with Yoshida the new parameter $\alpha=\sqrt{\lambda}$ and we get the following non-integrability result.

Proposition 5. If $e \notin\{-13,-3,-4 / 3,0,1,3\}$ then the Hamiltonian system defined by (7.3) has not a meromorphic first integral besides the Hamiltonian.

Proof. From the formulas (7.2) we compute the difference of exponents of the corresponding hypergeometric equation at the three singular points (see section 2) as $\hat{\lambda}=1-c=1 / 4,=\hat{\mu}=a-b= \pm \alpha / 2, \hat{\nu}=c-a-b=3 / 4$. The only cases of solvability are case (i) and case 5 of (ii) in Kimura's theorem, this gives us the following necessary conditions for integrability

(1) $\alpha=4 p$ or

(2) $\alpha=4 p \pm 1$ or

(3) $\alpha=4 p \pm 4 / 3$.

Now if the system is integrable, for a given value of $e$ we get two values of $\alpha$ and as one of these values is bounded (for $\alpha=\frac{3-e}{1+e}$ ), we only need to check a finite number of cases. The only possible cases are for the six values given in the proposition (we leave the details to the reader as an exercise).

ACKnowledgements. The authors are indebted to José Martinez-Alfaro who suggest to them the example of Sitnikov. They also are indebted to Haruo Yoshida who suggest to them the model of section 7 .

\section{REFERENCES}

[1] M. Alvarez and J. Delgado, The spring-pendulum system, in Hamiltonian Systems and Celestial Mechanics, E. A. Lacomba and J. Llibre, ed., World Scientific, Singapore, 1993.

[2] B. K. Berger, D. Garfinkle, and E. Strasser, New algorithm for Mixmaster dynamics, Classical and Quantum Gravity, 14 (1997), pp. L29-L36.

[3] F. Beukers, Differential Galois theory, in From Number Theory to Physics, W. Waldschmidt, P. Moussa, J.-M. Luck, and C.Itzykson, ed., Springer-Verlag, 1995, pp. 413-439.

[4] O. I. Bogoyavlensky, Qualitative Theory of Dynamical Systems in Astrophysics and Gas Dynamics, Springer, 1985.

[5] J. J. Cornish and J. J. Levin, Preprint, gr-qc/9605029.

[6] R. C. Churchill, J. Delgado, And D. L. Rod, The Spring-Pendulum System and the Riemann Equation, New Trends for Hamiltonian Systems and Celestial Mechanics, E. A. Lacomba and J. Llibre, ed., World Scientific, Singapore, 1996.

[7] R.Cushman and J. Sniatycki, Local integrability of the Mixmaster model, Reports on Mathematical Physics, 36 (1995), pp. 75-89. 
[8] A. Duval, The Kovacic algorithm with applications to special functions, in Differential Equations and Computer Algebra, M. Singer, ed., Academic Press, 1991, pp. 113-130.

[9] A. Duval and M. Loday-Richaud, A propos de l'algorithme de Kovacic, Applicable Algebra in Engineering, Communication and Computing, 3 (1992), pp. 211-246.

[10] I. Kaplansky, An Introduction to Differential Algebra, Hermann, 1976.

[11] T. KimurA, On Riemann's equations which are solvable by quadratures, Funkcialaj Ekvacioj, 12 (1969), pp. 269-281.

[12] J. J. KovACIC, An algorithm for solving second order linear homogeneous differential equations, J. Symb. Comput., 2 (1986), pp. 3-43.

[13] S. Kowalevski, Sur le probleme de la rotation d'un corps solide autour d'un point fixe, Acta Math., 12 (1889), pp. 177-232.

[14] L. Landau and E. Lifchitz, Théorie des champs, Mir, 1970.

[15] A. Latifi, M. Musette, And R. Conte, The Bianchi IX cosmological model is not integrable, Physics Letters A, 194 (1994), pp. 83-92.

[16] J. Martinet and J. P. Ramis, Théorie de Galois différentielle et resommation, in Computer Algebra and Differential Equations, E. Tournier, ed., Academic Press, 1989, pp. 117-214.

[17] J. Martinez-Alfaro and C. Chirald, Invariant Rotational Curves in the Sitnikov Problem, Celestial Mechanics and Dynamical Astronomy, 55 (1992), pp. 351-367.

[18] C. W. Misner, Mixmaster universe, Physical Review Letters, 20 (1969), pp. 1071-1074.

[19] J. J. Morales-Ruiz And J. P. Ramis, Galoisian obstructions to integrability of Hamiltonian systems, Methods and Applications of Analysis, 8 (2001), pp. 33-96.

[20] J. J. Morales-Ruiz And J. P. Ramis, A note on the non-integrability of some Hamiltonian systems with a homogeneous potential, Methods and Applications of Analysis, 8 (2001), pp. 113-120.

[21] J. Moser, Stable and Random Motions in Dynamical Systems, Princeton Univ. Press, Princeton, 1973.

[22] E. G. C. Poole, Introduction to the Theory of Linear Differential Equations, Oxford Univ. Press, 1936.

[23] M. F. Singer, An outline of differential Galois theory, in Computer Algebra and Differential Equations, E. Tournier, ed., Academic Press, 1989, pp. 3-57.

[24] M. F. Singer And F. Ulmer, Galois groups of second and third order linear differential equations, J. Symbolic Computation, 16 (1993), pp. 1-36.

[25] M. F. Singer AND F. Ulmer, Liouvillian and algebraic solutions of second and third order linear differential equations, J. Symbolic Computation, 16 (1993), pp. 37-73.

[26] A. H. TAuB, Empty space-times admitting a three parameter group of motions, Annals of Mathematics, 53 (1951), pp. 472-490.

[27] F. Ulmer And J. A. WeIL, Note on Kovacic's algorithm, J. Symbolic Computation, 22 (1996), pp. 179-200.

[28] H. Yoshida, On a class of variational equations transformable to the Gauss hypergeometric equation, Celestial Mechanics and Dynamical Astronomy, 53 (1992), pp. 145-150. 
